August 12, 2021

\title{
The Structure of Projected Center Vortices in Lattice Gauge Theory
}

\author{
R. Bertle ${ }^{a}$, M. Faber ${ }^{a}$, J. Greensite ${ }^{b, c}$, and $\check{S}$. Olejník ${ }^{d}$ \\ ${ }^{a}$ Inst. für Kernphysik, Technische Universität Wien, \\ A-1040 Vienna, Austria. E-mail: faber@kph.tuwien.ac.at \\ ${ }^{b}$ Physics and Astronomy Dept., San Francisco State Univ., \\ San Francisco, CA 94117, USA. E-mail: greensit@stars.sfsu.edu \\ $c$ Theory Group, Lawrence Berkeley National Laboratory, \\ Berkeley, CA 94720, USA. E-mail: greensit@lbl.gov \\ ${ }^{d}$ Institute of Physics, Slovak Academy of Sciences, \\ SK-842 28 Bratislava, Slovakia. E-mail: fyziolej@savba.sk
}

\begin{abstract}
We investigate the structure of center vortices in maximal center gauge of SU(2) lattice gauge theory at zero and finite temperature. In center projection the vortices (called Pvortices) form connected two dimensional surfaces on the dual four-dimensional lattice. At zero temperature we find, in agreement with the area law behaviour of Wilson loops, that most of the P-vortex plaquettes are parts of a single huge vortex. Small P-vortices, and short-range fluctuations of the large vortex surface, do not contribute to the string tension. All of the huge vortices detected in several thousand field configurations turn out to be unorientable. We determine the Euler characteristic of these surfaces and find that they have a very irregular structure with many handles. At finite temperature P-vortices exist also in the deconfined phase. They form cylindric objects which extend in time direction. After removal of unimportant short range fluctuations they consist only of space-space plaquettes, which is in accordance with the perimeter law behaviour of timelike Wilson loops, and the area law behaviour of spatial Wilson loops in this phase.
\end{abstract}




\section{Introduction}

It is very well known that the center symmetry is of crucial importance for QCD on the lattice. At the beginning of eighties it was shown that the deconfinement phase transition is connected with the spontaneous breaking of center symmetry [1]. In the confined phase field configurations are center symmetric, leading to symmetric distributions of Polyakov loops and to infinite energy of single quarks. In the deconfined phase one center element is favoured by the Polyakov loop distribution, quark charges can be screened by the gluon field.

There is now increasing evidence that center symmetry is not only relevant as an order parameter for confinement, but is also the crucial concept in understanding how confinement comes about. The idea that center vortices are responsible for confinement was put forward at the end of 70's [2 [6], but numerical evidence in favor of this idea is rather recent [7 11]. Our principle tool for locating vortices, and investigating their effect on gauge-invariant quantities, is "center projection" in maximal center gauge. Maximal center gauge is a gauge where all link variables are rotated as close as possible to center elements of the gauge group. Center projection is a mapping from the $\mathrm{SU}(2)$ link variables to $Z_{2}$ link variables. "P-vortices", formed from plaquettes with link product equal to -1 (we will call them P-plaquettes), are simply the center vortices of the projected $Z_{2}$ gauge-field configurations.

P-vortices on the projected lattice are thin, surface-like objects, while center vortices in the unprojected configuration should be surface-like objects of some finite thickness. Our numerical evidence indicates that $\mathrm{P}$-vortices on the projected lattice locate thick center vortices on the unprojected lattice. We also find that these thick vortices are physical objects, and that the disordering effect of such vortices is responsible for the entire QCD string tension. Details may be found in ref. [9]; a discussion of Casimir scaling in the context of the the center vortex theory is found in ref. [12] and [13].

In this article we discuss the structure of P-vortices in center-projected field configurations. In section two we show that P-vortices tend to form very large vortices (as required if they are the driving mechanism behind quark confinement, see also ref. [14), and that small-scale fluctuations of the vortex surfaces don't contribute to the string-tension. Further, we determine the orientability and the Euler characteristic of these vortices. In section three we investigate the structure of P-vortices at finite temperature. We find, in agreement with Langfeld et al. [15], that in the deconfined phase the vortices are oriented along timelike surfaces in the dual lattice, and are closed by the lattice periodicity; thereby explaining the differing behaviour of timelike and spacelike Wilson loops in this phase. 


\section{P-vortices at zero temperature}

\subsection{Finding P-vortices}

$\mathrm{P}$-vortices are identified by first fixing to the direct version of maximal center gauge, which in $\mathrm{SU}(2)$ gauge theory maximizes

$$
\sum_{x, \mu}\left|\operatorname{Tr} U_{\mu}(x)\right|^{2} .
$$

Then we map the $\mathrm{SU}(2)$ link variables $U_{\mu}(x)$ to $Z_{2}$ elements

$$
Z_{\mu}(x)=\operatorname{sign} \operatorname{Tr}\left[U_{\mu}(x)\right]
$$

The plaquettes with $Z_{\mu, \nu}(x)=Z_{\mu}(x) Z_{\nu}(x+\hat{\mu}) Z_{\mu}(x+\hat{\nu}) Z_{\nu}(x)=-1$ are the "P-plaquettes." The corresponding dual plaquettes, on the dual lattice, form the closed surfaces (in $\mathrm{D}=4$ dimensions) associated with P-vortices. This can be easily understood by constructing such a P-vortex out of a trivial field configuration with links $Z_{\mu}=1$. By flipping a single link to $Z_{\mu}=-1$ the six space-time plaquettes attached to this link form an elementary Pvortex. In dual space the corresponding plaquettes form the surface of a cube. By flipping neighbouring links in real space we get cubes in dual space attached to each other. The dual vortex is the connected surface of these cubes.

The distribution of these $\mathrm{P}$-vortices in space-time determines the string tension. The number $n$ of piercings of P-vortices through a Wilson loop determines the value of the projected Wilson loop $W_{c p}(I, J)$ of size $A=I \times J$

$$
W_{c p}(I, J)=(-1)^{n}
$$

If $p$ is the probability that a plaquette belongs to a P-vortex, then by assuming the independence of piercings we get for the expectation value of $W_{c p}(I, J)$

$$
\left\langle W_{c p}(I, J)\right\rangle=[(1-p) 1+p(-1)]^{A}=(1-2 p)^{A}=e^{-\sigma_{c p} A} \approx e^{-2 p A}
$$

where the string tension in center projection is

$$
\sigma_{c p}=-\ln (1-2 p) \approx 2 p
$$

However, for small vortices the independence assumption of piercings is not fulfilled, simply because one piercing is always correlated with another piercing nearby, and therefore small vortices in the average do not contribute to the area law fall-off of large Wilson loops. For this reason, confining vortex configurations will have to be very large, with an extension comparable to the size of the lattice. Fig. 11 shows the P-vortex plaquettes in a single timeslice of an equilibrium field configuration on a $12^{4}$-lattice at $\beta=2.3$.

The above defined probability $p$ scales nicely with the inverse coupling $\beta$. This was shown in refs. [9, 10]. However, already from Fig. 1 of ref. [7] it can be seen that the $\chi(1,1)$ 


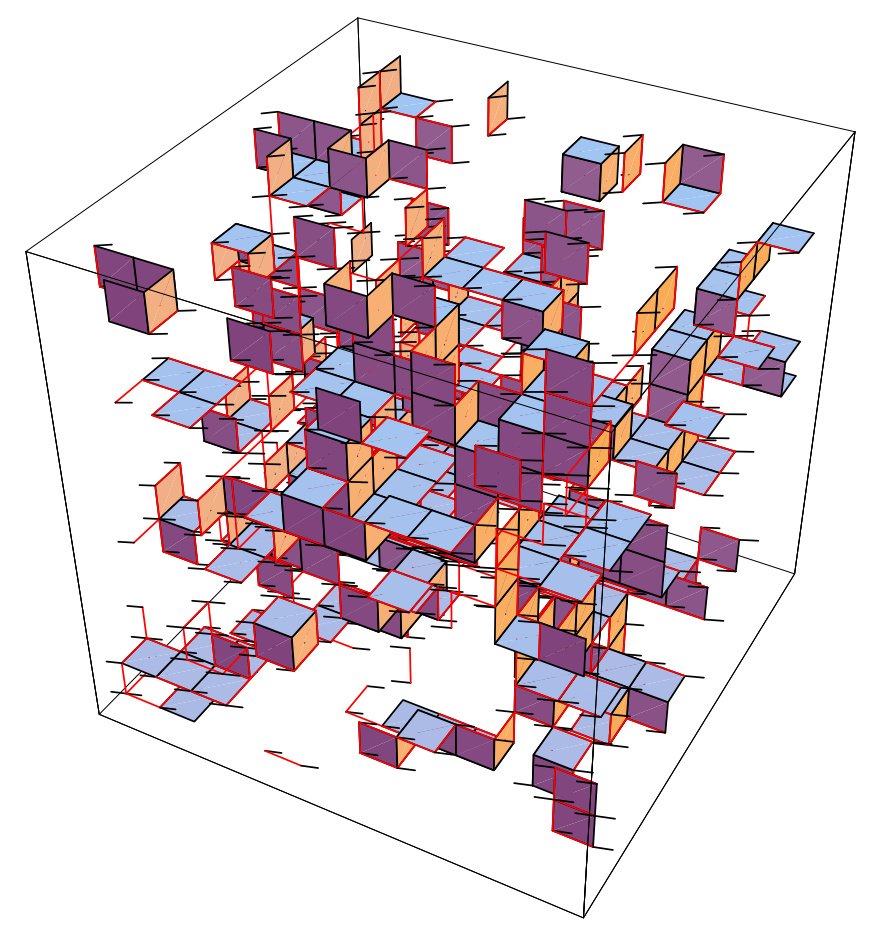

Figure 1: 3-dimensional cut through the dual of a $12^{4}$-lattice at $\beta=2.3$. P-plaquettes are indicated. They form closed two-dimensional surfaces. P-plaquettes which extend in time direction and connect the given 3-dimensional space with neighbouring time-slices are indicated by small amputated lines in forward or backward direction.

Creutz ratios lie above the asymptotic string tension $\sigma$, and therefore the values of $p$ come out higher than those of $f$ that can be inferred from $\sigma$ using

$$
f=\left(1-e^{-\sigma}\right) / 2
$$

The values of $p$ and $f$ are shown in Fig. 2 for various $\beta$-values. Here $p$ is denoted as "unsmoothed" (to stress the difference from values extracted using a smoothing procedure that will be introduced in section 2.2), while $f$ is denoted as "stringtension" in Fig. 目 and was extracted from $\chi(3,3)$ Creutz ratios of the center projected field configurations. As shown in ref. [12] the values of $\chi(3,3)$ are in good agreement with the determinations of the string tension by Bali et al. [16]. Other data sets shown in Fig. 2 denoted as " $n$-smoothing" will be discussed in section 2.2 .

There appears the interesting question about the difference between $p$ depicted in Fig. 2 under the name "unsmoothed" and $f$. Introducing various smoothing methods of $\mathrm{P}$-vortices we will show below that this difference originates in short distance fluctuations of P-vortex surfaces. 


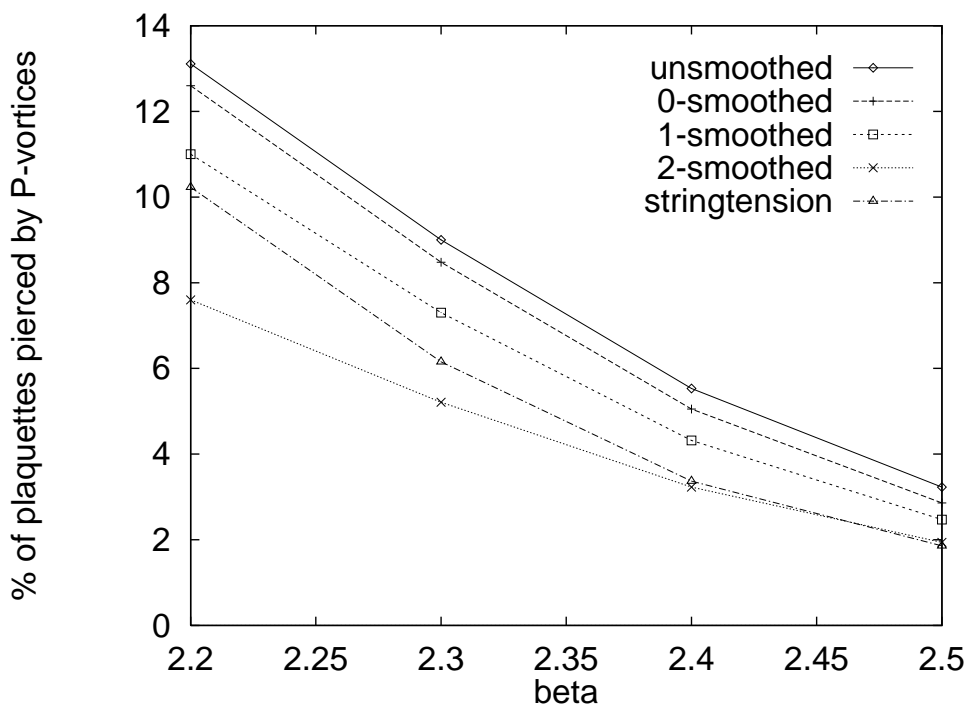

Figure 2: Percentage $p$ of $\mathrm{P}$-vortex plaquettes for various $\beta$-values and smoothing steps is compared with the fraction $f$ of plaquettes which should be pierced according to the asymptotic string tension $\sigma$.

\subsection{Size of P-vortices}

One of the most basic properties of vortices which is connected with the above raised questions is their size. In order to check the size and number of $\mathrm{P}$-vortices we determine neighbouring P-plaquettes, which can be easily done on the dual lattice. In most cases there is no doubt about the neighbouring plaquettes, since most dual links connect only two dual P-plaquettes. But in some cases there appear ambiguities, when dual links are attached to 4 or 6 dual P-plaquettes. Below we investigate these ambiguities in more detail and discuss possible resolutions.

In general P-vortices can be in contact at sites, links and plaquettes. Let's have a look at these different possibilities. A contact point is of no importance since we define the connectedness via common links. For our case of the $Z_{2}$ center group a plaquette belonging to two independent vortices leads to a fusion of these vortices. In this context, it is important to understand if the irregular structure of P-vortices depicted in Fig. 1 is due to such fusion process of simpler vortices. The remaining possibility, a contact of P-vortices at links needs a more detailed discussion.

A simple example of such field configurations are two cubes touching like in Fig. 3. For such a configuration in dual space it is not clear whether it builds one or two vortices. At the given length scale there is no unique solution for the question of connectedness. Connecting 1 with 2, 3 with 4 would result in one vortex, connecting 1 with 3,2 with 4 in two separated vortices. In most cases the situation can be resolved by postponing the decision about the connectedness of these plaquettes until, by following the vortex surface in all other directions, the indicated plaquettes (usually) turn out to be members of the same vortex. There appear some cases where no decision is possible by these means, as in 


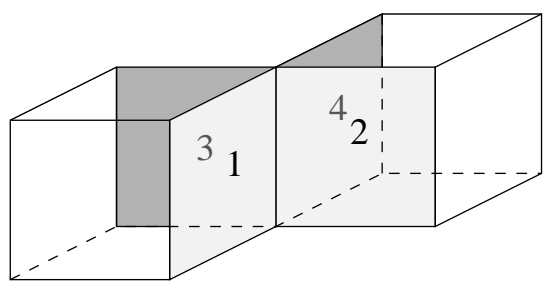

Figure 3: The question of connectedness can not be solved uniquely for this configuration of P-vortices.

the simple example of Fig. 3. In order to get a lower limit for the size of vortices we decide in such cases to treat the configuration as two separate vortices.

It may even occur that vortices touch along closed lines. In these cases parts of the vortex surface can't be reached following regular connections of plaquettes. These cases are even not so rare, their percentage is shown in Fig. A. The length of the closed line is usually very small and includes in the average 5 to 7 links.

With the above mentioned rules for deciding connectivity of P-vortices in ambiguous cases, we determine the P-vortex sizes. Since most of the plaquettes turn out to belong to the same vortex the most interesting vortex is the largest. The full line in Fig. 5 shows the percentage of $\mathrm{P}$-vortices belonging to the largest vortex for various values of $\beta$, see also [17]. For the evaluation at beta $=2.2,2.3,2.4$ and 2.5 we used 2000, 2000, 800 and 240 field-configurations on $12^{4}$-, $12^{4}$-, $16^{4}$ - and $22^{4}$-lattices, resp. It is obvious that for $T=0$ and all investigated $\beta$-values there is mainly one huge vortex, which contains around $90 \%$ of all P-plaquettes. All other vortices are rather small and should not contribute to the string tension according to the above given arguments. Small vortices means strongly correlated

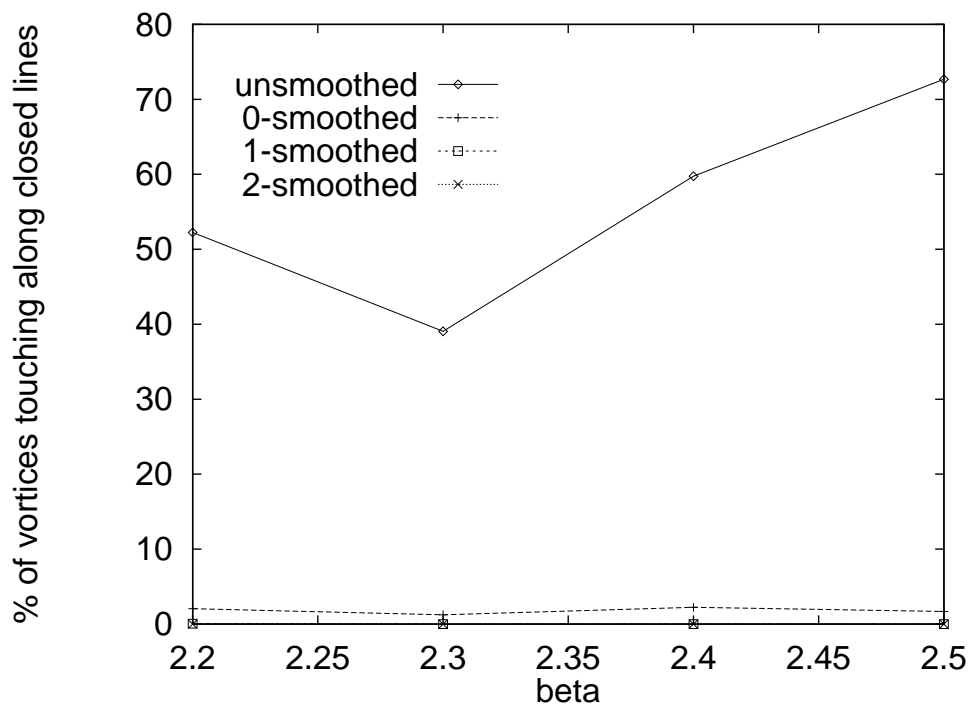

Figure 4: Percentage of such P-vortices which touch along closed lines for various types of smoothing. 


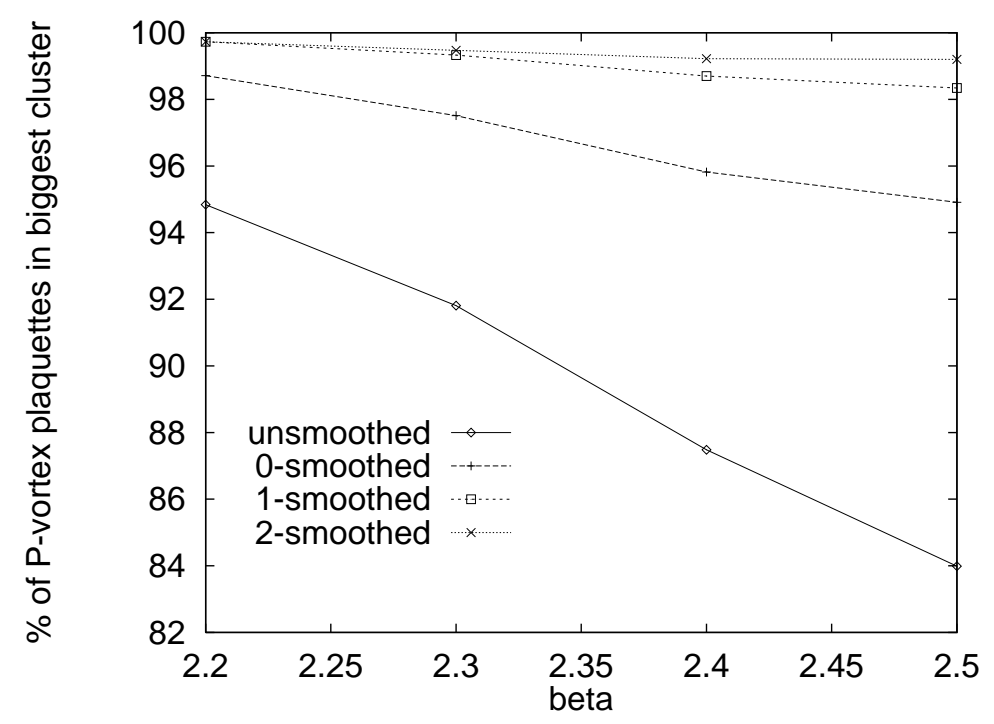

Figure 5: Percentage of P-plaquettes in the largest vortex for various types of smoothing.

piercings resulting in a perimeter contribution to Wilson loops. P-vortices of diameter $d$ lead to correlations for distances larger than $d$. Only vortices extending over the whole space contribute to an area law at all length scales. We conclude that the string tension is determined by the area of a single huge P-vortex.

The fact that $\mathrm{P}$-vortices in the confined phase have an extension comparable to the lattice size is a recent result of Chernodub et al. [14, and our finding is consistent with theirs. The fact that there appears to be only one very large vortex may also be related to results reported by Hart and Teper 18\|. These authors find that large monopole loops, identified in abelian projection, intersect to form one huge cluster. Since our previous studies [8] indicate that abelian-projection monopoles loops occur mainly on P-vortex surfaces, it seems quite natural that large loops on a single large surface would tend to intersect.

\subsection{Small-scale fluctuations of P-vortices}

In the preceding section we gave an argument why small vortices do not contribute to the string tension. By the same argument, small fluctuations of the vortex surface affect only perimeter law contributions. We will remove these short range fluctuations from P-vortex surfaces and show that the percentage of plaquettes belonging to such smoothed vortices gives directly the string tension $\sigma$.

In order to follow this idea we introduce several smoothing steps which are depicted in Fig. 6. In a first step we identify single isolated P-vortex cubes consisting of six dual P-plaquettes only and remove them. Since we substitute in this step 6 plaquettes by 0 we call this step 0 -smoothing. In the next step called 1-smoothing we identify cubes covered by 5 P-vortex plaquettes. Such cubes can be substituted by one complementary 


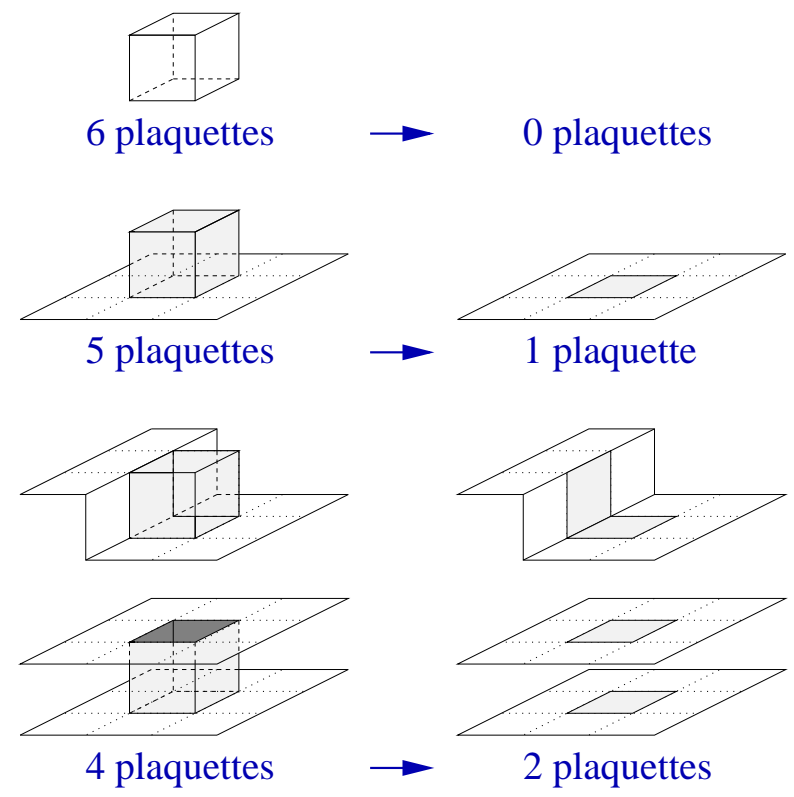

Figure 6: Various smoothing steps for vortices.

plaquette which closes the cubes. Finally, we substitute cubes with 4 plaquettes by the complementary 2 plaquettes. There are two different arrangements for these 4, resp. 2 plaquettes. In the 2-smoothing step we substitute both of them in accordance with Fig. 6 . In order to visualize the effect of smoothing on the appearance of vortices we show in Fig. 7 the result of 2-smoothing for the configuration in Fig. 11.

In Fig. 5 we compare for various smoothing steps the probability that a P-plaquette belongs to the largest vortex. It is clearly seen that the largest reduction in the number of vortex plaquettes is already achieved with 0 -smoothing. After 2 -smoothing the probability reaches more than $99 \%$ which shows that only very few small vortices survive the smoothing procedure. In all investigated field configurations we found a single huge P-vortex, we never met a configuration with two large vortices.

The relation between the percentage $p_{i}$ of P-plaquettes after $i$-smoothing and the fraction $f$ which one would expect from the physical string tension $\sigma$ according to Eq. 6 can be seen from Fig. 2. One can see that the value of $p_{i}$ nicely approach $f$, especially for the larger values of $\beta$ where the $\mathrm{P}$-plaquettes get less dense.

Further we check the Creutz ratios extracted from P-configurations after various smoothing steps. The results are shown in Fig. 8. It is clearly seen that 0 - and 1 -smoothing does not change the extracted Creutz ratios. At $\beta=2.2$, where the percentage of P-vortex plaquettes is of the order of $10 \%, 2$-smoothing causes some small $(\sim 5 \%)$ reduction in $\chi(3,3)$, but even this small deviation goes away at larger $\beta$.

Center projected Creutz ratios $\chi(R, R)$ are nearly independent of $R$. Only $\chi(1,1)$ (the logarithm of $1 \times 1$ loop) deviates from the Creutz ratios for large loops. This behaviour indicates that the only significant correlation among P-plaquettes is at a distance of one lattice spacing. The short-range fluctuations which may be responsible for this correlation 


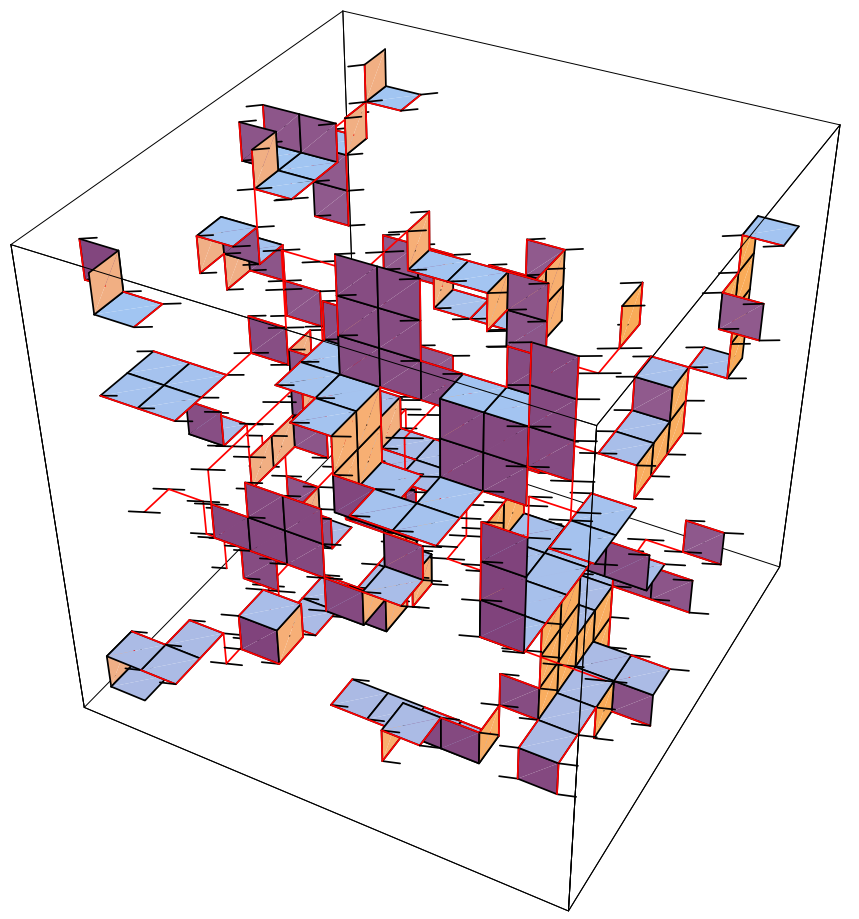

Figure 7: Result of the configuration of Fig. 1 after 2-smoothing. P-plaquettes which extend in time direction and connect the given 3-dimensional space with neighbouring time-slices are indicated by small amputated lines in forward or backward direction.

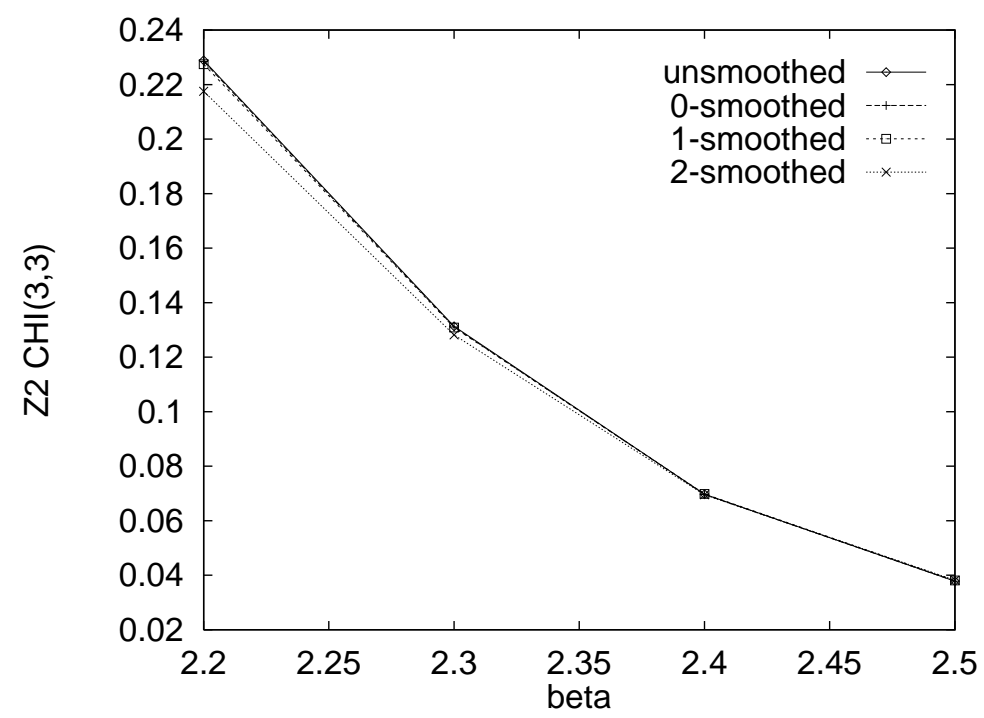

Figure 8: Creutz ratios for different smoothing steps. 
are removed by our smoothing procedure. When this is done, the values of $p$ and $f$ defined above come together, and the string tension is almost unchanged.

\subsection{Topological properties}

The basis for the investigation of the topology of P-vortices is the following rule: The type of homomorphy of a surface can be determined if it is connected, compact and closed. Then, it is determined by a) the orientation behaviour, b) the Euler characteristic. Pvortices in dual space would fulfill the requested conditions if every link joined only two plaquettes. As mentioned above this is not always the case.

Therefore, we will proceed in the following way. First, we will treat the determination of the orientability for the case that every link joins uniquely two attached P-vortex plaquettes. For each link of every P-plaquette we specify a sign. We start with an arbitrary P-plaquette and fix an arbitrary rotational direction. Those two links which are run through in positive axis direction get a plus sign, the other two get a minus sign. We continue at an arbitrary neighbouring P-plaquette. Its rotational sense we fix in such a way that the joining link gets the opposite sign than before. We continue this procedure for every plaquette of the given $\mathrm{P}$-vortex. If at the end every link of the $\mathrm{P}$-vortex has two opposite signs we call such $\mathrm{P}$-vortices orientable. The simplest example is a threedimensional cube. If some links appear with two equal signs the P-vortex is unorientable, e.g. with the topology of a Klein bottle.

We already gave a certain classification for those cases where four or six P-plaquettes are joined by a single link, see also Fig. 3. In cases of this kind, where different pairs of P-plaquettes can be treated as belonging to independent vortices, we determine the orientability as though the vortices did not touch at any link. With this procedure we get an upper bound for the orientability of vortices. Analogously we proceed for the case of a vortex touching itself at a link. We determine the orientability for a configuration where this touching is avoided.

The simulation shows that without exceptions the large vortices in all investigated field configurations, for all investigated $\beta$-values, turned out to be unorientable surfaces. We checked for the various employed smoothing steps whether this behaviour remains unchanged. It turns out that P-vortices remain unorientable after smoothing; apparently the smoothing procedure does not remove all of the local structures (e.g. "cross-caps") responsible for the global non-orientability.

The second property which determines the topological properties of P-vortices is the Euler characteristic $\chi$ which is defined by

$$
\chi=\mathcal{N}_{0}-\mathcal{N}_{1}+\mathcal{N}_{2},
$$

where $\mathcal{N}_{k}$ is the number of $k$-simplices: $\mathcal{N}_{0}$ is the number of vertices, $\mathcal{N}_{1}$ the number of links, and $\mathcal{N}_{2}$ the number of plaquettes. $\chi$ is directly related to the genus $g$ of a surface, in the orientable case by

$$
\chi=2-2 g
$$




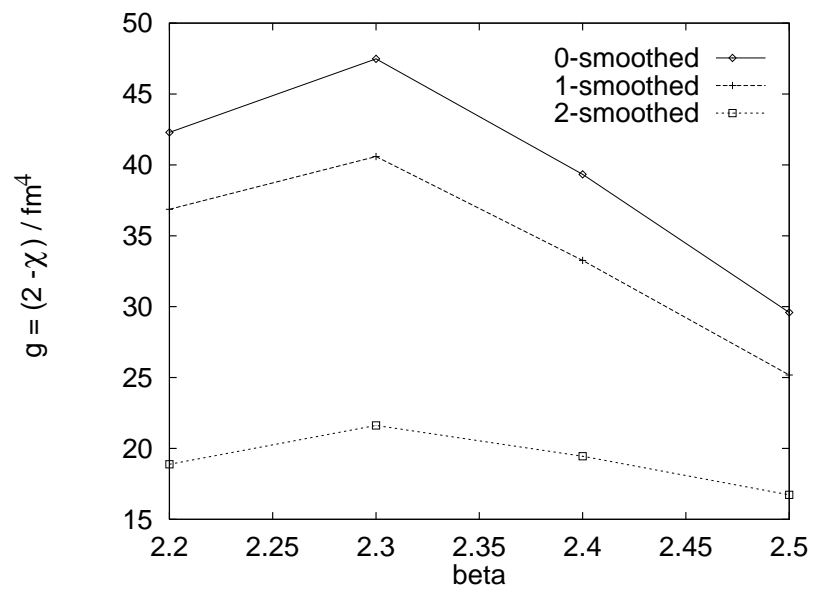

Figure 9: Genus $g$ of $\mathrm{P}$-vortices per $\mathrm{fm}^{4}$ for various inverse couplings $\beta$.

and in the unorientable case by

$$
\chi=2-g .
$$

An orientable surface of genus $g$ is homeomorphic to a sphere with $g$ attached handles. An unorientable surface of genus $g$ corresponds to a sphere with $g$ attached Möbius strips (also known as "cross-caps").

The determination of the Euler characteristic of a P-vortex is not inhibited by possible self-touchings. We can simply treat the vortex as it is; the result is the average between a possible separation and a real fusing of the two parts of the vortex. For a detailed discussion of this case, we refer the interested reader to ref. [17].

In Fig. 9 we show the genus $g=2-\chi$ per $\mathrm{fm}^{4}$ of P-vortices for various values of $\beta$. For the prediction of these values we used $\sqrt{\sigma} / \Lambda=58$ and $\sqrt{\sigma}=440 \mathrm{MeV}$. Without smoothing the genus takes a maximal value around $\beta=2.3$. With 0 -smoothing, only elementary vortices are removed and therefore $\chi$ is unchanged. With 1-smoothing, contact points and contact links can be removed, therefore a reduction of the genus of a vortex by 1 -smoothing is to be expected. In Fig. 9 this reduction is of the order of $15 \%$. By 2smoothing also regular bridges in vortices can be removed, decreasing in this way the genus. This reduction amounts to $55 \%$ at $\beta=2.2$ and $43 \%$ at $\beta=2.5$. After 2 -smoothing the genus stays more nearly constant with $\beta$ than without smoothing. It has to be investigated how the behaviour of $g$ behaves at still higher $\beta$-values. The trend in the investigated region seems compatible with a scaling behaviour for genus $g$, and is not compatible with a selfsimilar short-range structure below the confinement length scale. Fractal structure of that kind would lead to an increase of the genus with $\beta$, as more handles are uncovered at ever-shorter length scales. Of course, even a smoothed P-vortex surface will be rough at length scales beyond the confinement scale, and an appropriate fractal dimension can be defined. The fractal dimension of unsmoothed P-vortex surfaces, using the definition of dimension $D=1+2 A / L$, where $A$ is the number of plaquettes and $L$ the number of links on the vortex surface, has been reported in refs. [14] and [19]. 


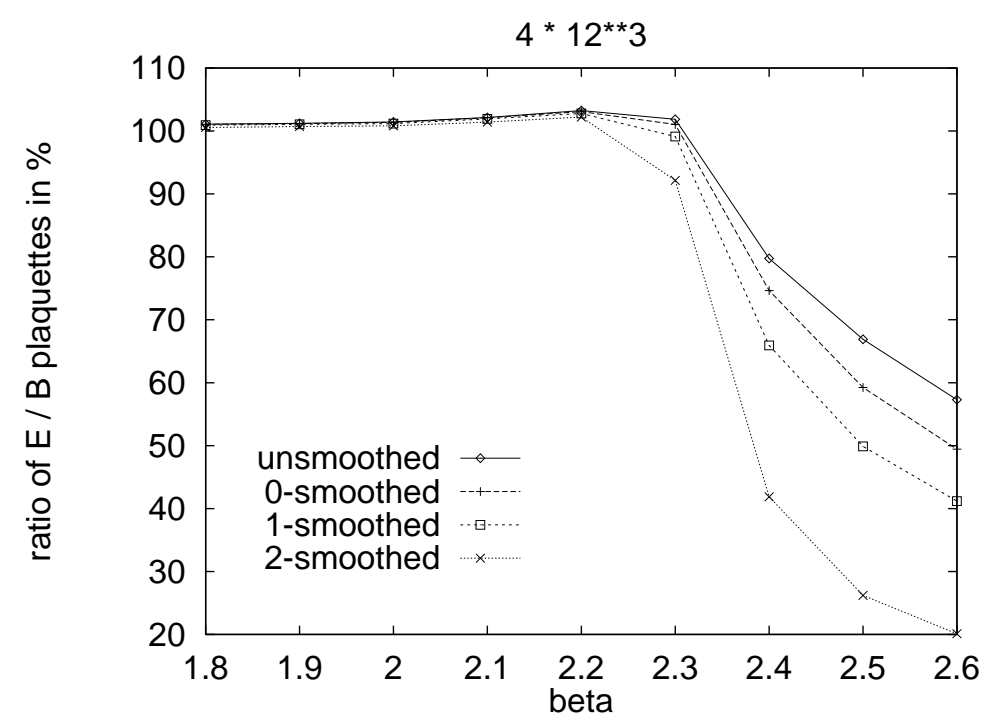

Figure 10: Ratio of space-time (E) and space-space (B) P-plaquettes as a function of $\beta$ for a $4 \cdot 12^{3}$-lattice.

These investigations of the topology of P-vortices show that they are not topologically 3 -spheres. This is not so surprising; there was no particular reason that vortices should have this topology. The structure which we identified, huge vortices extending over the whole lattice, unorientable with a lot of handles, is quite consistent with rotational symmetry in four dimensions. But as we will see, at finite temperature this symmetry can be destroyed.

\section{Topology of P-vortices at finite temperature}

The first discussion of the confinement/deconfinement phase transition in the context the vortex theory and center-projection methods, was made by Langfeld et al. in ref. [15], who give a nice explanation of the space-space string tension in the deconfined phase in terms of vortices closed in the time direction by lattice periodicity. Another very interesting investigation into the effect of finite temperature on vortex structure is due to Chernodub et al. 14. In this section we will extend our study of P-vortex topology, and the effect of our smoothing steps on $\mathrm{P}$-vortices, to the finite temperature case.

We did our finite temperature calculations on a $2 \cdot 12^{3}$-lattice for $\beta$-values between 1.6 and 2.6 and on a $4 \cdot 12^{3}$-lattice for $\beta$-values between 1.8 and 2.6. With a heat-bath-algorithm we measured after 1000 equilibration steps 1000 configurations with a distance of 20 for each investigated $\beta$-value.

The most striking difference to zero temperature calculations is the strong asymmetry of P-plaquette distributions in the deconfined phase which can be seen in Fig. 10. As a shortnotation we use E-plaquette for space-time and B-plaquette for space-space P-plaquettes. An investigation of this asymmetry was also performed in [15]. Just below the phase transition the density of E-plaquettes is slightly larger than the density of B-plaquettes. 


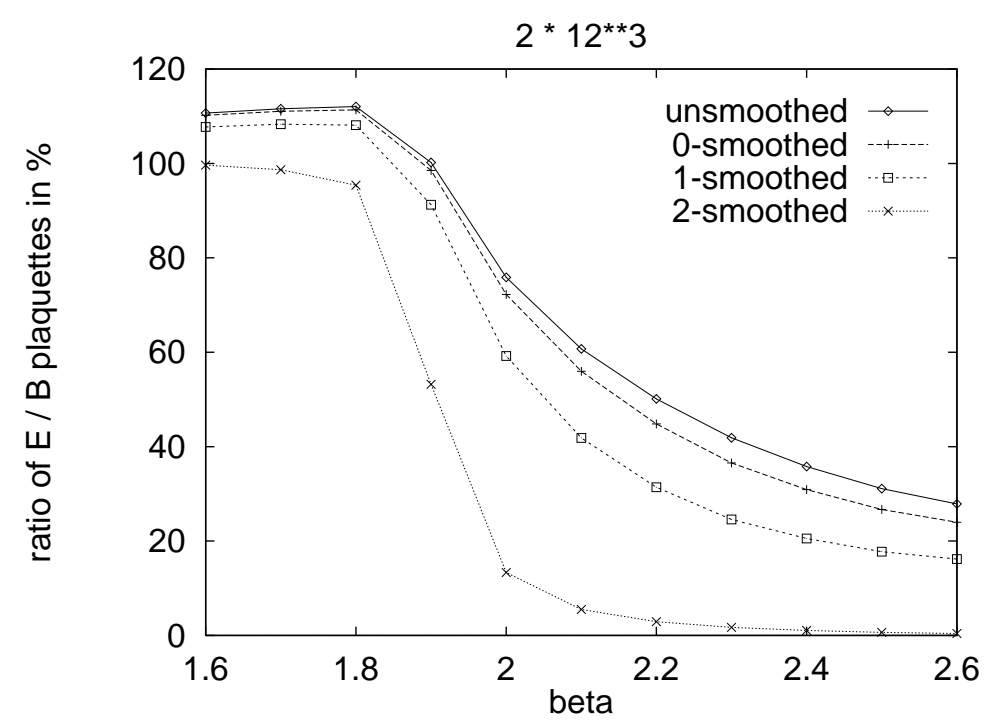

Figure 11: Same as Fig. 10 for a $2 \cdot 12^{3}$-lattice.

The excess is even larger for a time extent of 2 lattice units where it amounts to more than $10 \%$ as one can see in Fig. 11. The excess at $N_{t}=2$ seems to be connected with short range fluctuations of the vortices, since it is greatly reduced by smoothing. The detected strong asymmetry in the deconfined phase gives a very intuitive explanation for the behaviour of space-time and space-space Wilson loops, as previously discussed in ref. [15. The dominant vortex which percolates through the lattice is a (mostly) timelike surface on the dual lattice, which is closed via periodicity in the time direction. Polyakov lines are not affected by timelike vortex surfaces, and timelike Wilson loops are also unaffected. Therefore the string tension of timelike loops is lost in the deconfinement phase. On the other hand, large timelike vortex surfaces (composed of B-plaquettes) do disorder spacelike Wilson loops, which accounts for the string tension of spatial loops (c.f. [20]) in the deconfinement regime.

In the deconfined phase, the density of E-plaquettes is strongly decreasing with smoothing and for 2-smoothing soon reaches values close to $0 \%$ as seen in Figs. 10 and 11. Eplaquettes in the deconfined phase appear obviously due to short range fluctuations and can't contribute to an area law behaviour.

Fig. 12 displays the dual P-plaquettes of a typical field configuration at $\beta=2.6$ on a $2 \cdot 12^{3}$-lattice. The dual P-plaquettes form cylinders in time direction, closed via the periodicity of the lattice. Vortices of this shape are also well known in finite temperature theory under the name of ordered-ordered interfaces [21].

The density of P-plaquettes is depicted in Fig. 13 for various smoothing steps. The decrease in the number of $\mathrm{P}$-plaquettes in the 0 -smoothing step is almost independent of $\beta$. The number of $\mathrm{P}$-plaquettes above the phase transition decreases rather slowly.

As expected from the zero temperature results in the confined phase most of the Pplaquettes belong to a single large vortex. This can be seen in Fig. 14. The situation changes 


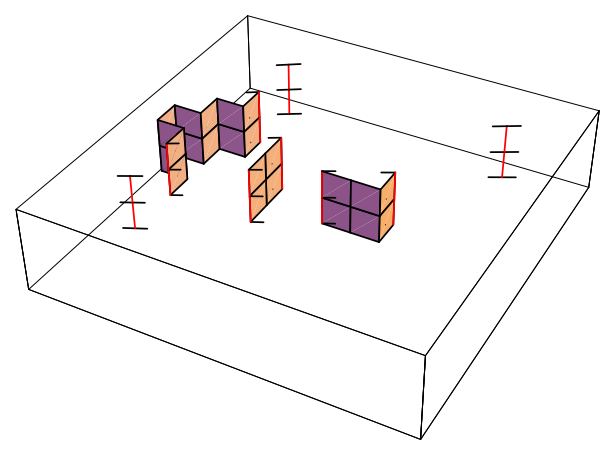

(a)

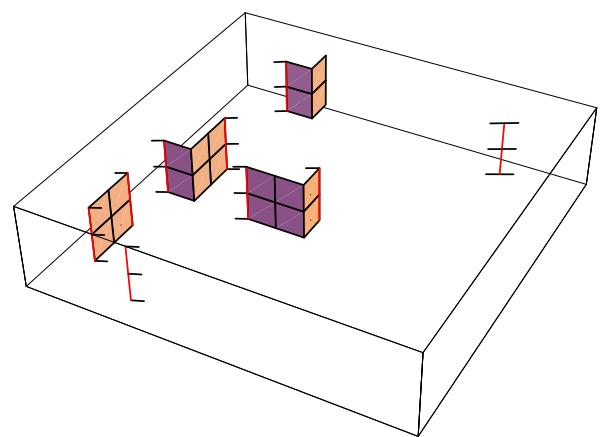

(b)

Figure 12: Dual P-plaquettes in a typical field configuration at $\beta=2.6$, on a $2 \cdot 12^{3}$-lattice. Two successive $\mathrm{z}$-slices for the $\mathrm{x}-\mathrm{y}$-t-subspace are shown. The amputated lines leaving the left figure towards right arrive in the right figure from the left.

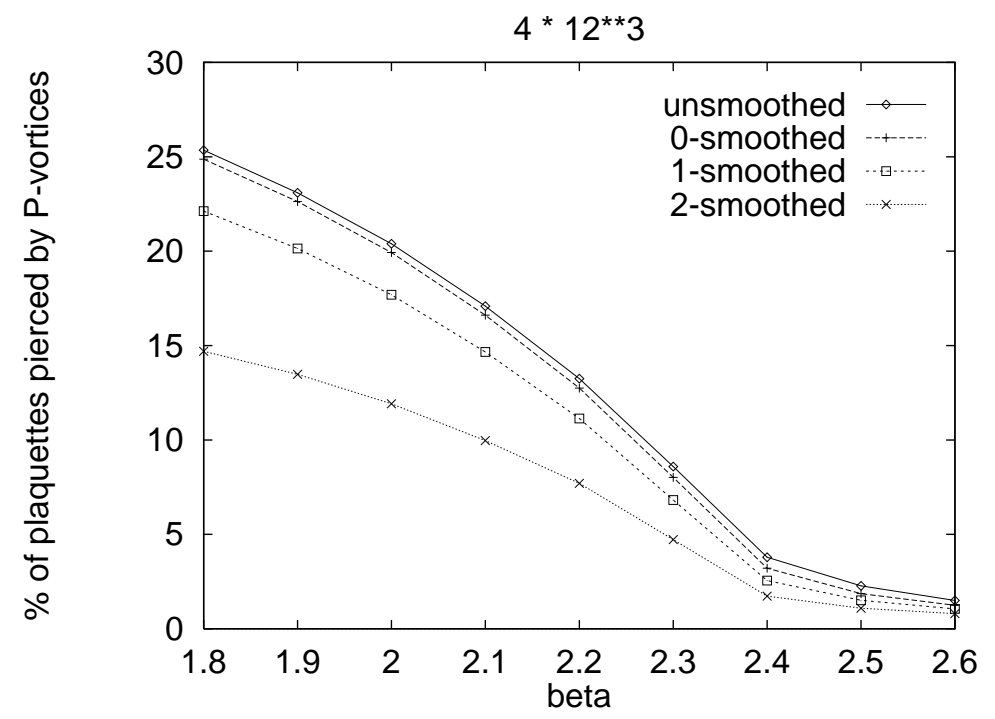

Figure 13: Percentage of P-plaquettes as a function of $\beta$ for a $4 \cdot 12^{3}$-lattice. 


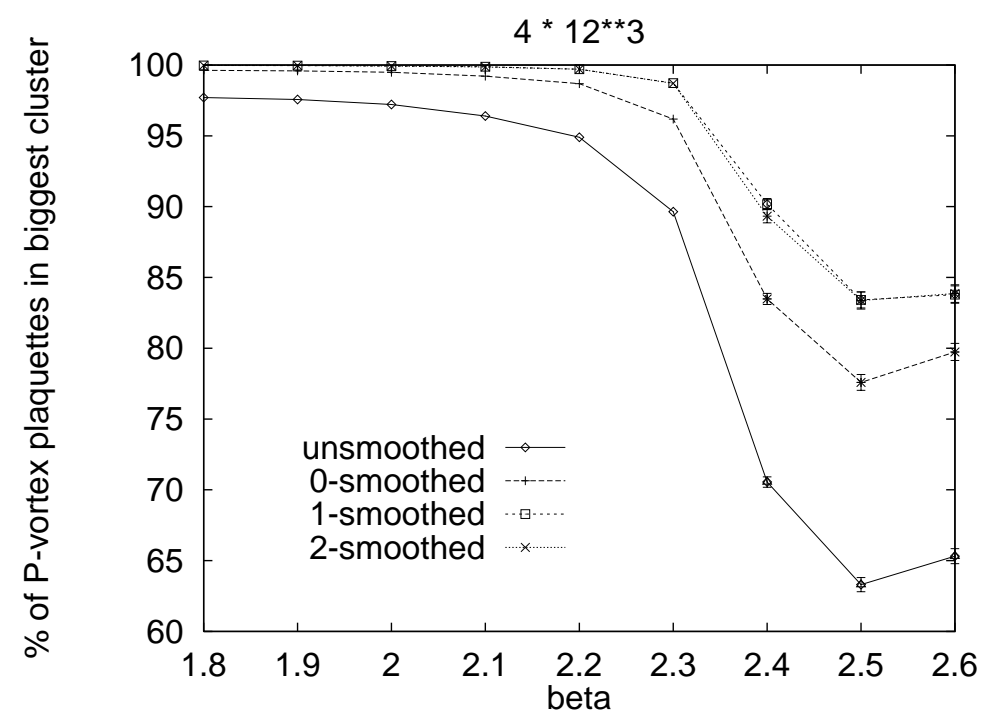

Figure 14: Size of largest vortex in \% of all P-plaquettes for a $4 \cdot 12^{3}$-lattice.

drastically at the phase transition where the percentage of P-plaquettes in the largest vortex drops considerably, especially for the unsmoothed configurations. The smoothing procedure shows that there is still one largest vortex but its dominance is not so strong as in the zero-temperature case. The increase of the percentage from $\beta=2.5$ to $\beta=2.6$ could be just a finite size effect; this will require investigation on larger lattices. In any case, the existence of a large space-time vortex on the dual lattice is required, at finite temperature in the deconfinement phase, in order to explain area law behaviour for spacelike Wilson loops.

With the decrease in the percentage of E-plaquettes we find increasing orientability of P-vortices in Fig. 15. The orientability approaches $100 \%$ for large $\beta$. The smoothing procedure shows which part of the unorientability is due to short range fluctuations of the vortex.

The relations between genus $g$ and Euler characteristic $\chi$ are different for orientable (8) and unorientable surfaces (9). Since both types of surfaces appear in finite temperature calculations we investigate the value of $2-\chi$ as in the zero-temperature case. For orientable vortices this expression is the genus $g$, for unorientable surfaces half of the genus. In Fig. 16 we show the value of $2-\chi$ for the largest vortex. These data are not scaled with the lattice constant $a$ as in the zero-temperature case (Fig. 9). In the confined phase P-vortices are again complicated surfaces and especially 2 -smoothing reduces the number of handles. Above the phase transition $2-\chi$ approaches the value 2. This is a consequence of the vanishing density of E-plaquettes. The largest P-vortex becomes orientable with genus $g=1$ and $\chi=0$. It has the topology of a torus. 


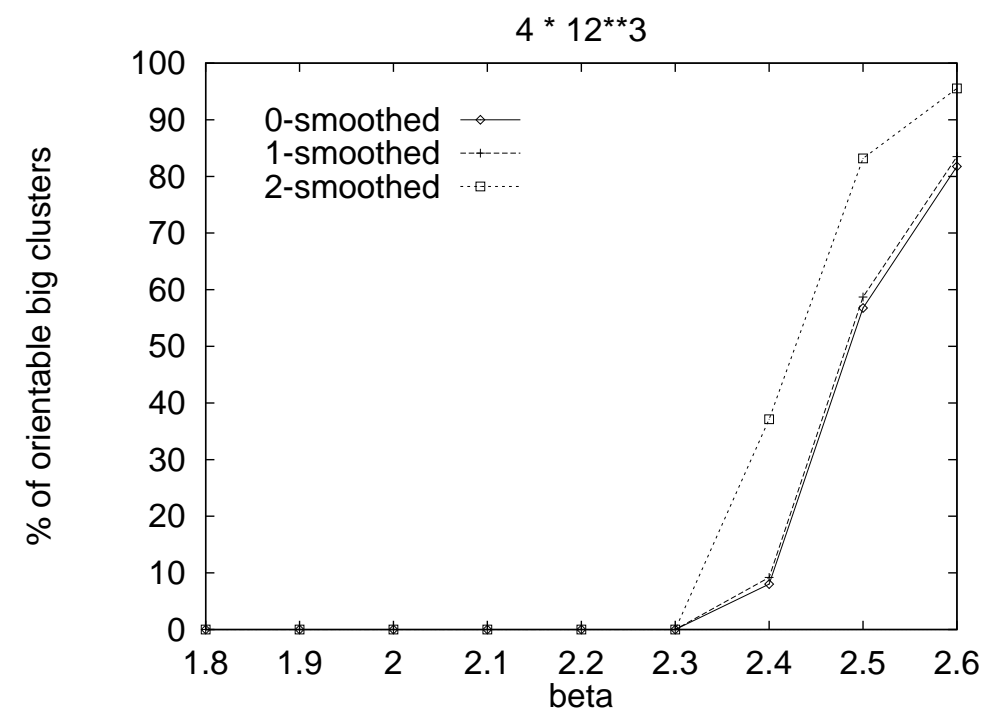

Figure 15: Percentage of orientable P-vortices for a $4 \cdot 12^{3}$-lattice.

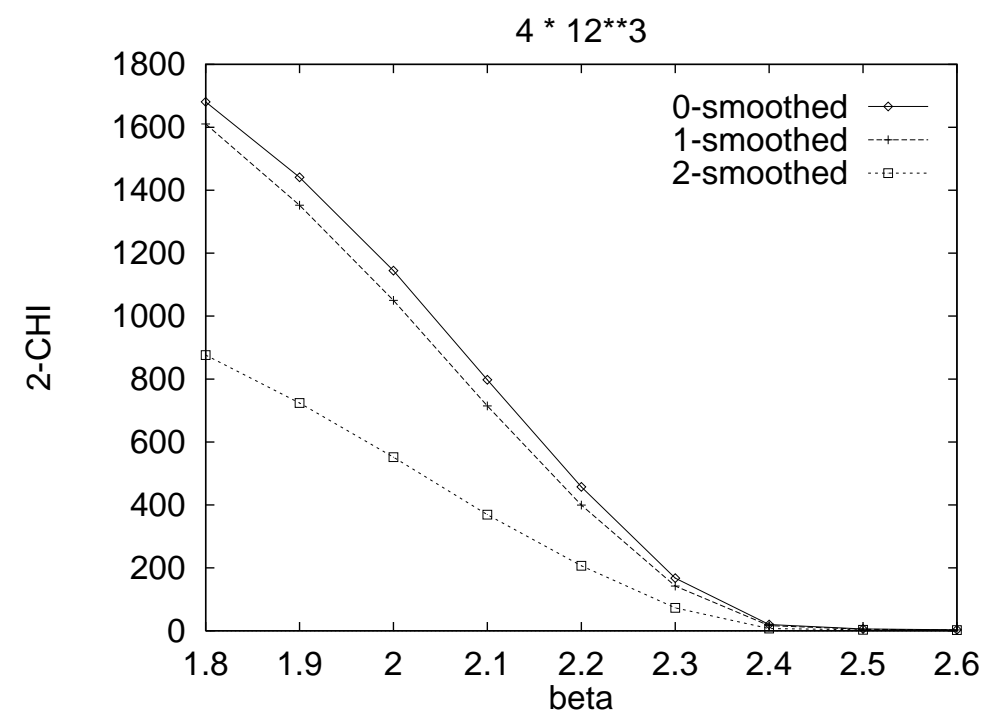

Figure 16: Genus of largest vortex for a $4 \cdot 12^{3}$-lattice. 


\section{Conclusions}

We have investigated the size and topology of P-vortices in $\mathrm{SU}(2)$ lattice gauge theory; P-vortices are surfaces in the dual lattice which lie at or near the middle of thick center vortices. We have found that in the confined phase the four-dimensional lattice is penetrated by a single huge P-vortex (see also [14]) of very complicated topology. This huge $\mathrm{P}$-vortex is a closed surface on the dual lattice which is unorientable and has many $\left(\sim 10 / \mathrm{fm}^{4}\right)$ handles. There exist also a few very small vortices. The short range fluctuations of the large P-vortex contribute only to the perimeter law falloff of projected Wilson loops. These short range fluctuations may simply be due to a slight ambiguity in the precise location of the middle of a thick center vortex, as was discussed in section 4 of ref. [9], and are not necessarily characteristic of the thick center vortices themselves.

By a smoothing procedure, we were able to remove these perimeter contributions due to short-range fluctuations, keeping the Creutz ratios constant. Thus the short-range P-vortex fluctuations are found to account for the difference between the percentage $p$ of plaquettes which are pierced by $\mathrm{P}$-vortices, and the comparatively smaller fraction $f$ which, in the simplest version of the vortex model (with uncorrelated P-plaquettes), contribute to the string tension. Upon smoothing away the short-range fluctuations, we find the fraction $p$ closely approaching the value of $f$ extracted from the asymptotic string tension.

The density of vortices does not vanish in the deconfined phase, but there is found to be a strong space-time asymmetry. P-vortices at finite temperature are mainly composed of space-space plaquettes forming timelike surfaces on the dual lattice. These surfaces are closed via the periodicity of the lattice in the time direction, they are orientable, and have the topology of a torus, i.e. genus $g=1$. The dominance of the largest vortex is not as strong as in the zero temperature case. The space-time asymmetry of P-vortices in the deconfined phase nicely explains [15] the corresponding asymmetry in Wilson loops, which have area-law falloff for spacelike, and vanishing string tension for timelike loops.

\section{Acknowledgements}

This work was supported in part by Fonds zur Förderung der Wissenschaftlichen Forschung P11387-PHY (M.F.), the U.S. Department of Energy under Grant No. DEFG03-92ER40711 (J.G.), the "Action Austria-Slovak Republic: Cooperation in Science and Education" (Project No. 18s41) and the Slovak Grant Agency for Science, Grant No. 2/4111/97 (̌̌.O.).

\section{References}

[1] J. Kuti, J. Polonyi, K. Szlachanyi, Phys. Lett. 98B (1981) 199.

[2] G. 't Hooft, Nucl. Phys. B153 (1979) 141.

[3] G. Mack, in Recent Developments in Gauge Theories, edited by G. 't Hooft et al. (Plenum, New York, 1980). 
[4] J. M. Cornwall, Nucl. Phys. B157 (1979) 392.

[5] H. B. Nielsen, P. Olesen, Nucl. Phys. B160 (1979) 380;

J. Ambjørn, P. Olesen, Nucl. Phys. B170 (1980) 60; 265.

[6] R. P. Feynman, Nucl. Phys. B188 (1981) 479.

[7] L. Del Debbio, M. Faber, J. Greensite, Š. Olejník, Phys. Rev. D55 (1997) 2298, heplat/9610005.

[8] L. Del Debbio, M. Faber, J. Greensite, Š. Olejník, in New Developments in Quantum Field Theory, ed. Poul Henrik Damgaard, Jerzy Jurkiewicz (Plenum Press, New YorkLondon, 1998) 47, hep-lat/9708023.

[9] L. Del Debbio, M. Faber, J. Giedt, J. Greensite, Š. Olejník, Phys. Rev. D58 (1998) 094501, hep-lat/9801027.

[10] M. Engelhardt, K. Langfeld, H. Reinhardt, O. Tennert, Phys. Lett. B419 (1998) 317, hep-lat/9710068.

[11] Ph. de Forcrand, M. D'Elia, hep-lat/9901020.

[12] M. Faber, J. Greensite, Š. Olejník, Phys. Rev. D57 (1998) 2603, hep-lat/9710039.

[13] M. Faber, J. Greensite, Š. Olejník, Acta Phys. Slov. 49 (1999) 177, hep-lat/9807008.

[14] M.N. Chernodub, M.I. Polikarpov, A.I. Veselov, M.A. Zubkov, hep-lat/9809158.

[15] K. Langfeld, O. Tennert, M. Engelhardt, H. Reinhardt, hep-lat/9805002.

[16] G. S. Bali, C. Schlichter, K. Schilling, Phys. Rev. D51 (1995) 5165, hep-lat/9409005.

[17] R. Bertle, Die Struktur projizierter Z2-Vortices in der gluonischen GitterQCD, diploma thesis at the Vienna University of Technology, March 1998, http://www.kph.tuwien.ac.at/bertle/pub/diplomathesis.

[18] A. Hart, M. Teper, Phys. Rev. D58 (1998) 014504, hep-lat/9712003.

[19] B.L.G. Bakker, A.I. Veselov, M.A. Zubkov, hep-lat/9902010.

[20] F. Karsch, E. Laermann, M. Lütgemeier, Phys. Lett. B346 (1995) 94, hep-lat/9411020.

[21] Dynamics of First Order Phase Transitions, ed. H.J. Herrmann, W. Janke, F. Karsch (World Scientific, Singapore, 1992). 